\title{
Predictive Factors of Mortality in Acute Aortic Dissection and Validity of the EuroSCORE Algorithm in a Small-Sized Cardiac Surgery Institution
}

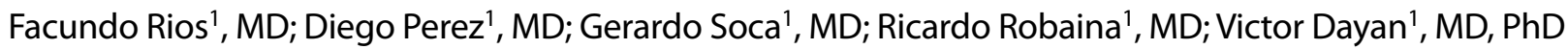

\begin{abstract}
Introduction: Acute aortic dissection (AAD) is a devastating surgical emergency, with high operative mortality. Several scoring algorithms have been used to establish the expected mortality in these patients. Our objective was to define the predictive factors for mortality in our center and to validate the EuroSCORE and Penn classification system.

Methods: Patients who underwent surgery for AAD from 2006 to 2016 were retrieved from the institution's database. Preoperative, operative and postoperative variables were collected. Observed and expected mortality was calculated by EuroSCORE. Logistic regression analysis and Cox regression analysis were performed to find predictors of operative mortality and survival, respectively. The receiver operating characteristic $(\mathrm{ROC})$ curves were plotted for logistic EuroSCORE, and the area under the ROC curve (AUC) was calculated.
\end{abstract}

Results: 87 patients $(27.6 \%$ female) underwent surgery for AAD. The mean age was $58.6 \pm 9.7$ years. Expected and observed operative mortality was $25.8 \pm 15.1 \%$ and $20.7 \%$, respectively. Penn $\mathrm{Aa}, \mathrm{Ab}$ and $\mathrm{Abc}$ shared similar observed/expected (O/E) mortality ratio. The only independent predictor of operative mortality (OR: 3.63; 95\% Cl: 1.19-11.09) and survival (HR: 2.6; 95\% Cl: 1.5-4.8) was female gender. EuroSCORE showed a very poor prediction capacity, with an AUC $=0.566$.

Conclusion: Female gender was the only independent predictor of operative mortality and survival in our institution. EuroSCORE is a poor scoring algorithm to predict mortality in AAD, but with consistent results for Penn $\mathrm{Aa}, \mathrm{Ab}$ and $\mathrm{Abc}$.

Keywords: ROC Curve. Aneurysm, Dissecting. Postoperative Period. Coronary Artery Bypass.

\begin{tabular}{ll}
\hline Abbreviations, acronyms \& symbols \\
\hline AAD & $=$ Acute aortic dissection \\
ACP & $=$ Antegrade cerebral perfusion \\
AMI & $=$ Acute myocardial infarction \\
AUC & $=$ Area under the curve \\
CI & $=$ Confidence interval \\
CPB & $=$ Cardiopulmonary bypass \\
HR & $=$ Hazard ratio \\
IRAD & $=$ International Registry of Acute Aortic Dissection \\
IRB & $=$ Institutional Review Board \\
OR & $=$ Odds ratio \\
ROC & $=$ Receiver operating characteristic \\
STS & $=$ Society of Thoracic Surgeons \\
TIA & $=$ Transient ischemic attack \\
\hline
\end{tabular}

${ }^{1}$ National Institute of Cardiac Surgery, Montevideo, Uruguay.

This study was carried out at the National Institute of Cardiac Surgery, Montevideo, Uruguay.

\section{INTRODUCTION}

Acute aortic dissection (AAD) is a surgical emergency. Despite quick diagnosis and surgery, it has a high operative mortality, which ranges between $19 \%$ and $32 \%{ }^{[1,2]}$. The explanation is mainly driven by its associated complications, such as cardiac tamponade, acute myocardial infarction (AMI), stroke and malperfusion syndrome.

Several authors have searched for predictors of mortality in patients with AAD. The Penn classification groups patients according to the presence of localized or general ischemia, among other things $s^{[3]}$. Scant data exists regarding the use of current scoring algorithms in small-sized cardiac surgery institutions.

Our aim was to evaluate predictors of mortality in patients with $A A D$ and to try to validate the Penn classification and EurOSCORE in our center.
Correspondence Address:

Victor Dayan

(iD) https://orcid.org/0000-0002-5470-0585

26 de Marzo 3459/602, Montevideo, Uruguay - Zip Code: 11300

E-mail: victor_dayan@hotmail.com 


\section{METHODS}

The Institutional Review Board (IRB) accepted the study and patient informed consent was waived. Data from the institution's database was searched from 2006 until 2016 to identify patients who underwent surgery for AAD. No exclusion criteria was used. Baseline, operative and postoperative variables were extracted.

Cardiovascular risk factors, clinical characteristics and physical examination at the time of presentation were identified. Expected mortality was assessed according to EuroSCORE I, which is the only validated score in our country. Additionally, we calculated the time from the onset of pain until surgery. Patients were classified according to the Penn classification: Aa - absence of vessel malperfusion or circulatory collapse; $A b$ - branch vessel malperfusion with ischemia; Ac - circulatory collapse with or without cardiac involvement; $\mathrm{Abc}$ - branch vessel malperfusion and circulatory collapse ${ }^{[3]}$.

Operative mortality was defined as mortality within 30 days of surgery or during initial hospital admission.

\section{Surgical Technique}

Midline sternal approach was used in all patients. Arterial cannulation was performed through the femoral, axillary artery or ascending aorta. Temperature during circulatory arrest varied according to the use of antegrade cerebral perfusion (ACP). Deep hypothermic $\left(18^{\circ} \mathrm{C}\right)$ circulatory arrest was performed in cases in which no ACP was used and moderate hypothermia in cases

Table 1. Baseline characteristics of the included patients.

\begin{tabular}{l|c}
\hline Variable & \\
\hline Age & $58.6(9.7)$ \\
\hline Female & $24(27.6)$ \\
\hline Hypertension & $67(77.9)$ \\
\hline Diabetes & $6(7.1)$ \\
\hline Tobacco use & $33(37.9)$ \\
\hline Previous MI & $4(4.6)$ \\
\hline Previous cardiac surgery & $4(4.6)$ \\
\hline Stroke & $3(3.4)$ \\
\hline PVD & $1(1.1)$ \\
\hline Creatininemia & $1.06(0.55)$ \\
\hline LVEF (\%) & $58.9(7.7)$ \\
\hline Bicuspid aortic valve & $9(10.3)$ \\
\hline EuroSCORE I & $25.8(15.1)$ \\
\hline LVEF & \\
\hline
\end{tabular}

LVEF=left ventricular ejection fraction; Ml=myocardial infarction; PVD=peripheral vascular disease in which it was used $\left(22-28^{\circ} \mathrm{C}\right)$. A composite conduit (St Jude Medical) was used in cases in which dissection or aneurysmal dilatation involved the aortic root. In these cases, we performed a Bentall-De Bono procedure with coronary ostial reattachment. If only the ascending aorta were involved, we would replace the affected segment with a prosthetic conduit. The aortic valve was resuspended with 3 pledgeted non-absorbable sutures in each of the commissures or replaced in cases in which regurgitation was more than moderate. The aortic arch was explored in all cases during circulatory arrest to evaluate the presence of additional tears.

\section{Statistics}

Continuous variables were expressed as mean \pm SD. Categorical variables were expressed as absolute value and percentages. Student's t-test or Mann-Whitney test was used to compare continuous variables. Categorical variables were compared using chi-square test. Survival was analyzed with Kaplan-Meier curves and compared with log-rank test. The receiver operating characteristic (ROC) curves were plotted for the logistic EuroSCORE, and the area under the ROC curve (AUC) was calculated as an index for the predictive value of the model.

\section{RESULTS}

From 2006 to 2016, 87 patients with AAD were operated on at our center. Mean age was $58.6 \pm 9.7$ years and $27.6 \%$ were female (Table 1). Bicuspid aortic valve was present in $10.3 \%$ of patients.

Regarding the clinical presentation, $71.3 \%$ complained of chest pain and $12.6 \%$ of dorsal pain. Complications at the time of clinical evaluation were present in $27 \%$ of patients (Table 2 ). Of these, the most frequent was distal ischemia. Considering the time elapsed from initial pain onset to surgery, $21.8 \%$ of the patients underwent surgery in $<24 \mathrm{~h}, 56.3 \%$ between $24-48 \mathrm{~h}$ and 10.3\% between 48-72h.

Patients who underwent surgery $<24 \mathrm{~h}$ had a higher incidence of complications (52.6\% vs. 29.4\%, $P=0.06$ ) and cardiac tamponade $(26.3 \%$ vs. $5.9 \%, P=0.01)$. The mean time from symptom onset to surgery in patients with complicated AAD was 20.8h $\pm 19.6 h$, significantly lower than in patients without complications (34.1 $\mathrm{h} \pm 32.7 \mathrm{~h}, \mathrm{P}=0.02)$.

Penn Aa was the most common presentation (72.4\%).

The most frequent surgical procedure performed was isolated replacement of the ascending aorta (78.2\%) (Table 3). The aortic valve was replaced in 19 patients (21.8\%). The mean duration of cardiopulmonary bypass (CPB) was $173 \pm 52$ minutes and circulatory arrest was performed in $55.2 \%$ of cases, with a mean duration of $35 \pm 11$ minutes. Cannulation of the femoral artery was the most frequent approach (49\%) and the lowest temperature obtained was $18.7 \pm 7.1^{\circ} \mathrm{C}$.

Expected operative mortality according to EuroSCORE was $25.8 \pm 15.1 \%$ and the observed mortality was $20.7 \%$. Operative mortality was higher in female patients $37.5 \%$ vs. $14.3 \%$, $P=0.014)$ and in patients who underwent surgery $<24 \mathrm{~h}(21.1 \% \mathrm{vs}$. $12.1 \%, P=0.049)$. Operative mortality was similar in patients who required aortic valve replacement (10.5\%) and those who did not 
Table 2. Clinical presentation of patients with acute aortic dissection $(n=87)$.

\begin{tabular}{|c|c|}
\hline Variable & \\
\hline \multicolumn{2}{|l|}{ Pain localization } \\
\hline Anterior chest & $62(71.3)$ \\
\hline Dorsal & $11(12.6)$ \\
\hline \multicolumn{2}{|l|}{ Complications } \\
\hline Tamponade & $3(3.4)$ \\
\hline Distal ischemia & $5(5.4)$ \\
\hline Shock & $3(3.4)$ \\
\hline Stroke & $1(1.1)$ \\
\hline Lower limb ischemia & $1(1.1)$ \\
\hline AMI & $2(2.3)$ \\
\hline More than 1 complication & $10(10.3)$ \\
\hline \multicolumn{2}{|l|}{ Pain onset to surgery } \\
\hline$<24 \mathrm{~h}$ & $19(21.8)$ \\
\hline 24-48h & $49(56.3)$ \\
\hline $48-72 h$ & $9(10.3)$ \\
\hline$>72 \mathrm{~h}$ & $10(11.4)$ \\
\hline \multicolumn{2}{|l|}{ Penn classification } \\
\hline $\mathrm{Aa}$ & $63(72.4)$ \\
\hline $\mathrm{Ab}$ & $4(4.6)$ \\
\hline Ac & $15(17.2)$ \\
\hline$A b c$ & $5(5.7)$ \\
\hline
\end{tabular}

$\mathrm{AMI}=$ acute myocardial infarction

(23.5\%, $P=0.216)$. Observed/expected mortality was quite similar and constant in all Penn types, except for Penn Ac (Table 4).

The most frequent complication was transient ischemic attack (TIA) (18.4\%). Mechanical assisted ventilation and ICU stay were 59.6 \pm 130.7 minutes and 6.3 \pm 12.1 days, respectively (Table 5).

Univariate regression analysis found only female gender (OR: 3.6; 95\% Cl: 1.2-10.7) and the lowest hematocrit in CPB (OR: 0.89; $95 \% \mathrm{Cl}$ : 0.80-0.99) as predictors of operative mortality. Circulatory
Table 3. Intraoperative characteristics of patients with acute aortic dissection.

\begin{tabular}{l|c}
\hline Variables & \\
\hline AA replacement & $67(78.2)$ \\
\hline AA + valve replacement & $15(17.2)$ \\
\hline Composite valve conduit & $4(4.6)$ \\
\hline CPB time & $173(52)$ \\
\hline Circulatory arrest (\%) & $55.2(63.2)$ \\
\hline Femoral cannulation & $49(56.3)$ \\
\hline Axillary cannulation & $14(16.1)$ \\
\hline Aortic cannulation & $24(27.5)$ \\
\hline AXC time (min) & $92(32)$ \\
\hline Circulatory arrest time & $35(11)$ \\
\hline Lowest temperature & $18.7(7.1)$ \\
\hline Hct pre-CPB (\%) & $35.7(8.6)$ \\
\hline Hct during CPB (\%) & $24.1(4.9)$ \\
\hline Hct post-CPB (\%) & $26.1(5.5)$ \\
\hline AA ascendingorta AxC & \\
\hline
\end{tabular}

$A A=$ ascending aorta; $A X C=$ aortic cross-clamp; $\mathrm{CPB}=$ cardiopulmonary bypass

arrest (OR: 3.6; 95\% Cl: 0.9-13.7) and surgery within 24 hours of symptom onset (OR: 3.0; 95\% Cl: 9.43-0.97), respectively, were at the borderline of statistical significance.

Comparison of baseline and operative variables between female and male patients showed that female patients had significantly lower hematocrit in CPB $(21.9 \pm 4.6 \%)$ than male patients (24.9 $\pm 4.8 \%, P=0.014)$. After multivariate regression analysis, only female gender resulted as an independent predictor for operative mortality (OR: 3.63; 95\% Cl: 1.19-11.09).

Five- and ten-year survival were $52.8 \pm 5.5 \%$ and $37.3 \pm 6.2 \%$, respectively (Figure 1). Female patients had significant worse survival (HR: 2.6; 95\% Cl: 1.5-4.8), even after excluding operative mortality (HR: 2.4; 95\% Cl: 1.1-5.3) (Figure 2).

Regarding the predictive value of the EuroSCORE I (logistic) algorithm, the ROC showed an AUC of 0.566 (Figure 3).

Table 4. EuroSCORE and observed operative mortality in patients according to the Penn classification.

\begin{tabular}{c|c|c|c|c}
\hline & Penn Aa & Penn Ab & Penn Ac & Penn Abc \\
\hline EuroSCORE (SD) & $25.7(14.2)$ & $37.2(23.1)$ & $22.7(14.7)$ & $26.4(21.2)$ \\
\hline Operative mortality (\%) & $11(17.5)$ & $1(25)$ & $5(33.3)$ & $1(20)$ \\
\hline O/E & 0.68 & 0.67 & 1.45 & 0.75 \\
\hline
\end{tabular}


Table 5. Postoperative outcomes in patients with acute aortic dissection.

\begin{tabular}{l|c}
\hline Variables & \\
\hline Total bleeding (ml) & $1604(1610)$ \\
\hline Days in ICU & $6.3(12.1)$ \\
\hline Mechanical ventilation (h) & $59.6(130.7)$ \\
\hline Days to discharge & $13.2(13.7)$ \\
\hline ARF & $39(44.8)$ \\
\hline Dialysis & $9(10.3)$ \\
\hline Stroke & $10(11.5)$ \\
\hline TIA & $16(18.4)$ \\
\hline Operative mortality (\%) & $18(20.7)$ \\
\hline
\end{tabular}

$\mathrm{ARF}=$ acute renal failure; $\mathrm{TI} A=$ transient ischemic attack

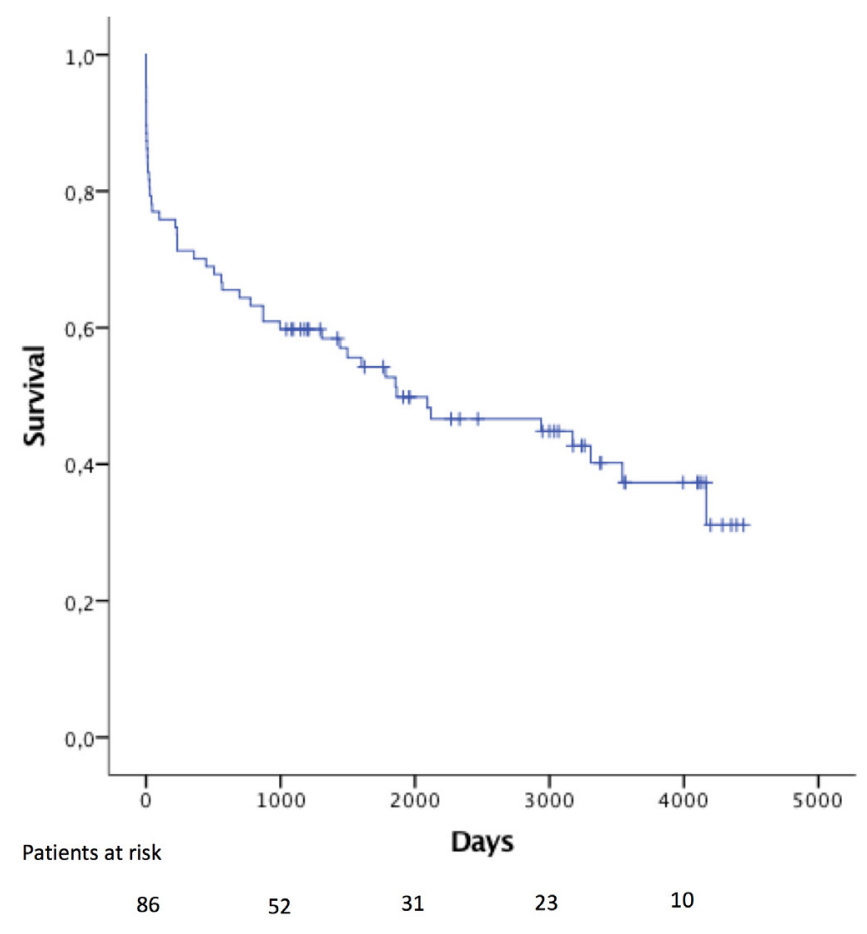

Fig. 1 - Overall survival in patients with $A A D$.

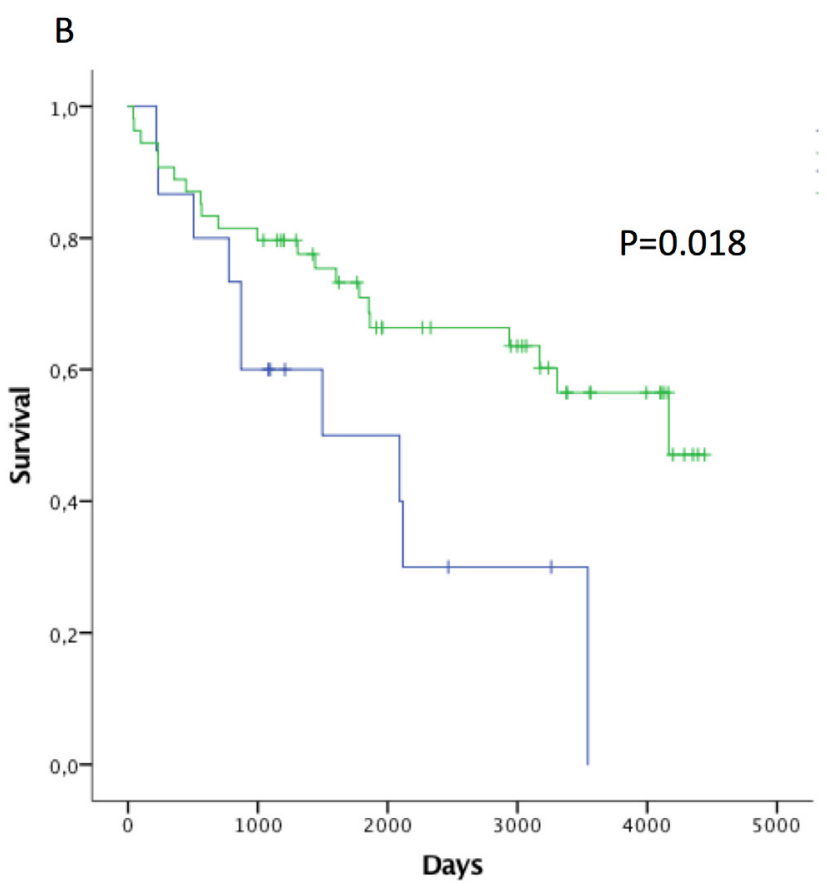

Patients at risk

$\begin{array}{lllrrr}\text { Female } & 14 & 9 & 5 & 2 & 0 \\ \text { Male } & 53 & 43 & 26 & 21 & 9\end{array}$

Fig. 2 - Survival according to gender before (A) and after (B) excluding operative mortality. 


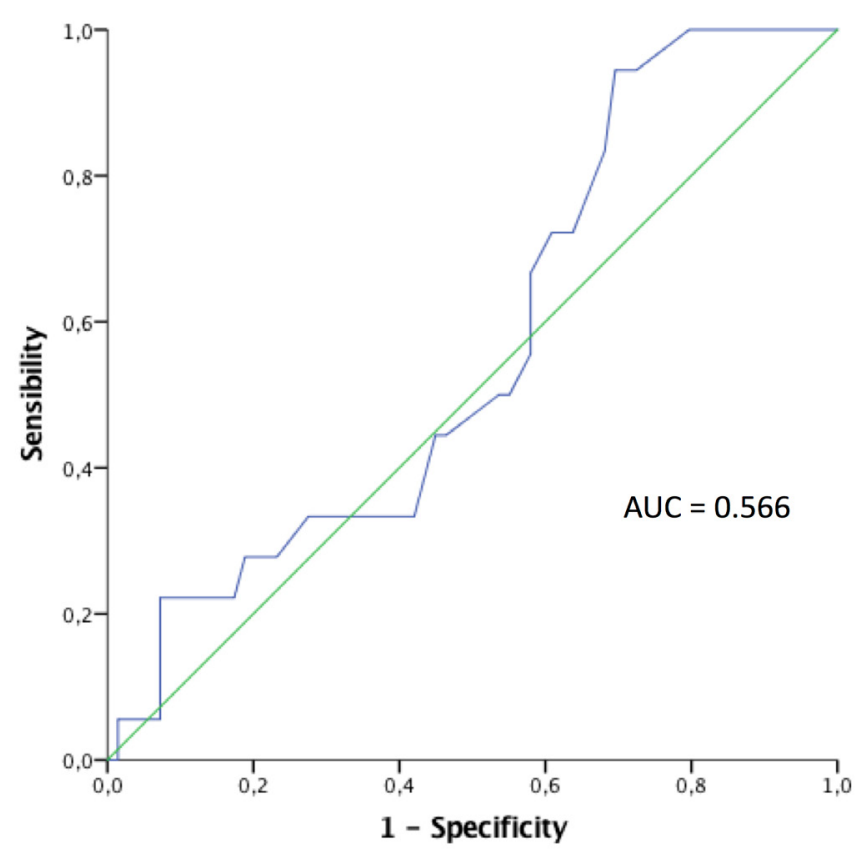

Fig. 3 - ROC analysis and AUC determination for EuroSCORE in operative mortality.

\section{DISCUSSION}

We found that AAD is associated with high operative mortality and low survival, similarly to other published reports ${ }^{[2]}$. We were able to show that the observed mortality was lower than the expected mortality according to EuroSCORE. Furthermore, the only predictor of mortality and long-term survival was female gender. EuroSCORE was not a useful algorithm to predict mortality, especially in patients with Penn Ac classification.

The International Registry of Acute Aortic Dissection (IRAD) has previously shown that female patients have increased mortality ${ }^{[4]}$. This difference in mortality between genders was seen especially in the 66-75-year-old group. In their study, Nienaber et al. ${ }^{[4]}$, reported $32 \%$ of operative mortality in female patients, which is similar to our data (37.5\%). In contrast to these authors, we found no differences in time from pain onset and surgery, as well as the incidence in the complication rate between male and female patients. The main difference in our study was a lower hematocrit during CPB in female patients, which was related in the univariate analysis to mortality. This lower hematocrit could probably be explained by a lower body surface area in female patients and, therefore, greater hemodilution with the initiation of CPB. Similar to Gokalp et al. ${ }^{[5]}$, the arterial cannulation approach was not a predictor of mortality.
Patients operated on within 24 hours of symptom onset had a higher incidence of complications associated with AAD and cardiac tamponade, which could explain the higher mortality in this group. Delaying surgery after 24 hours may lead to exclude patients with serious complications who die before arriving to the hospital and, therefore, bringing into play natural selection ${ }^{[6]}$. The time from symptom onset to surgery in complicated and non-complicated patients in our series was lower than that reported by Trimarchi et al. ${ }^{[7]}$ (62.9 hours and 105.1 hours for unstable and stable patients, respectively). Similar to Trimarchi et al. ${ }^{[7]}$, patients with complicated AAD underwent surgery at a significantly shorter time-lapse from the symptom onset. It is classically accepted that untreated patients with AAD have an associated mortality rate of $1 \%$ to $2 \%$ per hour immediately after symptom onset ${ }^{[8]}$. We support this statement and promote surgery as quick as possible after diagnosis. We believe the cause of surgery delay in our series is mainly due to the difficulty in establishing the diagnosis of AAD due to the lower incidence of complications.

Penn type Aa was the most frequent presentation followed by Penn abc. Similar to Augoustides et al. ${ }^{[3]}$, mortality was lower in patients without any ischemic complications. When we related our observed mortality to the expected mortality, we found the O/E ratio was quite constant among Penn groups, except in Penn Ac. In patients with circulatory collapse (Penn Ac), EuroSCORE underestimated the expected mortality. This is probably since the score is not sensitive enough for this group of patients. The predictive value of EuroSCORE was quite low in our study group. The AUC was well below 0.75 , which limits its role as a predictive algorithm for operative mortality in patients with AAD. Nishida et al. ${ }^{[9,10]}$ reported reliable prediction in 2006 using logistic EuroSCORE, but in 2014 found limited use of this scoring system. After the implementation of EuroSCORE I[ ${ }^{[11]}$, Howell et al. ${ }^{[11]}$ found that EuroSCORE II did not improve the prediction of mortality in high-risk patients. There is no defined data regarding the best risk score prediction model for AAD. Therefore, we believe that each center must evaluate the validity in AAD of its most used prediction algorithm and make use of all available tools in doing so.

\section{Limitations}

The small number of patients inherent in our small center does not allow us to make further associations nor firmly conclude on our findings. Furthermore, it does not allow us to create our own scoring algorithm. The only validated score for cardiac surgery in our country is the EuroSCORE I, therefore, we do not have data regarding the EuroSCORE II or Society of Thoracic Surgeons (STS) score in the included patients to ascertain its validity in AAD.

\section{CONCLUSION}

Female gender was the only predictor for operative mortality and long-term survival. Taking into consideration the Penn classification and the EuroSCORE, our data suggest that, although EuroSCORE is a poor algorithm to predict mortality, its prediction model is consistent with Penn Aa, Ab and Abc. 


\section{No financial support.}

\section{No conflict of interest.}

\section{Authors' roles \& responsibilities}

FR Substantial contributions to the conception or design of the work; or the acquisition, analysis or interpretation of data for the work; final approval of the version to be published

DP Substantial contributions to the conception or design of the work; or the acquisition, analysis or interpretation of data for the work; final approval of the version to be published

GS Substantial contributions to the conception or design of the work; or the acquisition, analysis or interpretation of data for the work; final approval of the version to be published

RR Substantial contributions to the conception or design of the work; or the acquisition, analysis or interpretation of data for the work; final approval of the version to be published

VD

Substantial contributions to the conception or design of the work; or the acquisition, analysis or interpretation of data for the work; final approval of the version to be published

\section{REFERENCES}

1. Olsson C, Hillebrant CG, Liska J, Lockowandt U, Eriksson P, FrancoCereceda A. Mortality in acute type A aortic dissection: validation of the Penn classification. Ann Thorac Surg. 2011;92(4):1376-82. doi:10.1016/j. athoracsur.2011.05.011.

2. Mehta RH, Suzuki T, Hagan PG, Bossone E, Gilon D, Llovet A, et al. Predicting death in patients with acute type a aortic dissection. Circulation. 2002;105(2):200-6. doi:10.1161/hc0202.102246.
3. Augoustides JG, Geirsson A, Szeto WY, Walsh EK, Cornelius B, Pochettino $A$, et al. Observational study of mortality risk stratification by ischemic presentation in patients with acute type A aortic dissection: the Penn classification. Nat Clin Pract Cardiovasc Med. 2009;6(2):140-6. doi:10.1038/ncpcardio1417.

4. Nienaber CA, Fattori R, Mehta RH, Richartz BM, Evangelista A, Petzsch M, et al. Gender-related differences in acute aortic dissection. Circulation. 2004;109(24):3014-21. doi:10.1161/01.CIR.0000130644.78677.2C.

5. Gokalp O, Yilik L, Iner H, Yesilkaya NK, Besir Y, Iscan S, et al. Comparison of femoral and axillary artery cannulation in acute type A aortic dissection surgery. Braz J Cardiovasc Surg. 2020;35(1):28-33. doi:10.21470/16789741-2018-0354.

6. Bachet J. Acute type A aortic dissection: can we dramatically reduce the surgical mortality? Ann Thorac Surg. 2002;73(3):701-3. doi:10.1016/ s0003-4975(02)03393-3.

7. Trimarchi S, Nienaber CA, RampoldiV, Myrmel T, Suzuki T, Mehta RH, et al. Contemporary results of surgery in acute type A aortic dissection: the international registry of acute aortic dissection experience. JThorac Cardiovasc Surg. 2005;129(1):112-22. doi:10.1016/j.jtcvs.2004.09.005.

8. Evangelista A, Isselbacher EM, Bossone E, Gleason TG, Eusanio $M D$, Sechtem $U$, et al. Insights from the international registry of acute aortic dissection: a 20-year experience of collaborative clinical research. Circulation. 2018;137(17):1846-60. doi:10.1161/ CIRCULATIONAHA.117.031264.

9. Nishida T, Masuda M, Tomita Y, Tokunaga S, Tanoue Y, Shiose A, et al. The logistic EuroSCORE predicts the hospital mortality of the thoracic aortic surgery in consecutive 327 Japanese patients better than the additive EuroSCORE. Eur J Cardiothorac Surg. 2006;30(4):578-82; discussion 582-3. doi:10.1016/j.ejcts.2006.07.011.

10. Nishida T, Sonoda H, Oishi Y, Tanoue Y, Nakashima A, Shiokawa Y, et al. The novel EuroSCORE II algorithm predicts the hospital mortality of thoracic aortic surgery in 461 consecutive Japanese patients better than both the original additive and logistic EuroSCORE algorithms. Interact Cardiovasc Thorac Surg. 2014;18(4):446-50. doi:10.1093/icvts/ivt524.

11. Howell NJ, Head SJ, Freemantle N, van der Meulen TA, Senanayake E, Menon A, et al. The new EuroSCORE II does not improve prediction of mortality in high-risk patients undergoing cardiac surgery: a collaborative analysis of two European centres. Eur J Cardiothorac Surg. 2013;44(6):1006-11; discussion 1011. doi:10.1093/ejcts/ezt174. 